\title{
Inefficacy of vancomycin and teicoplanin in eradicating and killing Staphylococcus epidermidis biofilms in vitro
}

\author{
J. Claessens ${ }^{\mathrm{a}, *}$, M. Roriz ${ }^{\mathrm{a}}$, R. Merckx ${ }^{\mathrm{a}}$, P. Baatsen ${ }^{\mathrm{b}}$, L. Van Mellaert ${ }^{\mathrm{c}}$, J. Van Eldere ${ }^{\mathrm{a}}$ \\ ${ }^{a}$ KU Leuven-University of Leuven, Department of Microbiology and Immunology, Laboratory of Clinical Bacteriology and Mycology, \\ UZ Leuven Campus Gasthuisberg, Herestraat 49, B-3000 Leuven, Belgium \\ ${ }^{\mathrm{b}}$ Electron Microscopy Facility (EMoNe) of the VIB BIO Imaging Core at KU Leuven-University of Leuven, Center for Human Genetics, Herestraat 49, \\ B-3000 Leuven, Belgium \\ ${ }^{\mathrm{c}}$ KU Leuven-University of Leuven, Department of Microbiology and Immunology, Laboratory of Molecular Bacteriology, Rega Institute for Medical \\ Research, Herestraat 49, B-3000 Leuven, Belgium
}

\section{A R T I C L E I N F O}

\section{Article history:}

Received 3 May 2014

Accepted 24 November 2014

\section{Keywords:}

Vancomycin

Teicoplanin

Biofilm biomass

Killing efficacy

Staphylococcus epidermidis

Biofilm

\begin{abstract}
A B S T R A C T
Biofilm-associated bacteria display a decreased susceptibility towards antibiotics. Routine assessment of antibiotic susceptibility of planktonic bacteria therefore offers an insufficient prediction of the biofilm response. In this study, in vitro biofilms of eight clinical Staphylococcus epidermidis strains were subjected to treatment with vancomycin, teicoplanin, oxacillin, rifampicin and gentamicin. In addition, the biofilms were subjected to combinations of an antibiotic with rifampicin. The effects on the biofilms were assessed by crystal violet staining to determine the total biofilm biomass, staining with XTT to determine bacterial cell viability, and microscopy. Combining these methods showed that treatment of S. epidermidis biofilms with glycopeptides increased the total biofilm biomass and that these antibiotics were not effective in killing bacteria embedded in biofilms. The decreased killing efficacy was more pronounced in biofilms produced by strains that were classified as 'strong' biofilm producers. Rifampicin, oxacillin and gentamicin effectively killed biofilm-associated bacteria of all tested strains. Combining antibiotics with rifampicin increased the killing efficacy without influencing the total biofilm biomass. When vancomycin or teicoplanin were combined with rifampicin, the increase in biofilm biomass was neutralised and also the killing efficacy was influenced in a positive way. We conclude that the combined methodology used in this study showed that glycopeptides were not effective in eradicating S. epidermidis biofilms but that combination with rifampicin improved the killing efficacy in vitro.
\end{abstract}

(c) 2015 Elsevier B.V. and the International Society of Chemotherapy. All rights reserved.

\section{Introduction}

Determination of the minimum inhibitory concentration (MIC) of an antibiotic is the standard method to determine bacterial susceptibility towards antibiotics. This method is based on the inhibition of planktonic growth of bacteria. However, in many types of infections, bacteria with a sessile lifestyle resulting in biofilms are involved [1,2]. A biofilm is defined as a multicellular aggregate of micro-organisms attached to a surface and embedded in a self-produced extracellular matrix. Bacteria growing in biofilms are characterised by increased resistance towards antibiotics and the host's immune response [2]. This is attributed to: (i) a different phenotype of bacteria growing in a biofilm compared with planktonic

\footnotetext{
* Corresponding author. Tel.: +32 16346 274; fax: +32 16346275.

E-mail addresses: jolien_cl@hotmail.com, jolien.claessens@med.kuleuven.be (J. Claessens).
}

bacteria, with decreased activity of the basic metabolic pathways and increased expression of stress response genes [3]; (ii) the extracellular matrix, which creates a micro-environment that interferes with antibiotic activity by decreasing penetration of the antibiotic, sequestering it or by the presence of modifying enzymes [4]; and (iii) the facilitated exchange of antibiotic resistance genes when bacteria are growing in high numbers close to each other [5].

Because of the decreased sensitivity of biofilm-associated bacteria to antibiotics, methods to evaluate antibiotic susceptibility should, in the case of biofilm-related infections, be supplemented with tests conducted on in vitro biofilms [6]. Different methods have been proposed to do this, including determination of the minimum antibiotic concentrations that inhibit biofilm growth (biofilm MIC), reduce the bacterial load of the biofilm [biofilm minimum bactericidal concentration $(\mathrm{MBC})]$ or completely eradicate the biofilm [minimum biofilm eradication concentration (MBEC)] [7]. However, the resulting antibiotic concentrations exceeded physiologically achievable concentrations [7]. Another way to determine 
antibiotic response consists of assessing the effects on the bacterial viability and the extracellular matrix separately [6]. In the present study, the effects of antibiotics on in vitro Staphylococcus epidermidis biofilms were evaluated using XTT staining as a measure for bacterial viability [8] and crystal violet (CV) to determine the total biofilm biomass [9]. These methods were combined with microscopic techniques to visualise the observed effects. The applied antibiotic concentrations corresponded to the peak serum concentrations, which is different from previously published studies that used subinhibitory antibiotic concentrations [10,11].

\section{Materials and methods}

\subsection{Bacterial strains and growth conditions}

Table 1 summarises the $S$. epidermidis strains used in this study. All strains, except S. epidermidis 567, were isolated from bloodstream infections associated with central venous catheters (CVCs). S. epidermidis 567 was isolated from a urinary tract catheter infection; this strain was kindly provided by Prof. Dr W. Ziebuhr (University of Würzburg, Würzburg, Germany) [12]. S. epidermidis 1457 is a type strain often used in biofilm research [13], and $S$. epidermidis $10 \mathrm{~b}$ was isolated from a confirmed case of catheter infection in the University Hospital Leuven (Belgium) [14]. In addition to the laboratory strains, clinical isolates from proven biofilm-related infections were used. These strains are designated S. epidermidis 12c, 13c, 16c, 17c and 22c (Table 1 ).

Tryptone soya broth (TSB) (Oxoid Ltd., Basingstoke, UK) was prepared according to the manufacturer's instructions. Growth conditions were adjusted to optimise biofilm formation and detection (Table 1). Because of the high biofilm formation capacities of S. epidermidis $1457,10 \mathrm{~b}$ and $22 \mathrm{c}$, biofilm formation of these strains was allowed to occur in 10\% TSB in phosphate-buffered saline (PBS) ( $\mathrm{pH}$ 7.2). This was done to adjust the total biofilm biomass to the upper detection limit of the enzyme-linked immunosorbent assay (ELISA) reader (VICTOR3 ${ }^{\mathrm{TM}}$ Multilabel Plate Reader; PerkinElmer Singapore Pte Ltd., Singapore). S. epidermidis 567 was characterised as a biofilm-negative, ica-positive strain; biofilm formation was induced by adding $4 \% \mathrm{NaCl}$ to the culture medium [15].

The antibiotics used to treat the biofilms were all purchased from Sigma-Aldrich Chemie GmbH (Steinheim, Germany). Antibiotic stock solutions, except rifampicin, were prepared in sterilised Milli- $Q{ }^{\circledR}$ water (Merck Millipore, Carrigtwohill, Ireland) and were kept at $-20^{\circ} \mathrm{C}$. The rifampicin stock was prepared in methanol and were kept at $-20^{\circ} \mathrm{C}$.

\subsection{Minimum inhibitory concentration determination}

The MICs of antibiotics towards planktonic bacteria were determined by the broth dilution method according to the European Committee on Antimicrobial Susceptibility Testing (EUCAST) [16]. Bacterial growth was assessed by measurement of the optical density at $590 \mathrm{~nm}$ using a VICTOR3 ${ }^{\mathrm{TM}}$ Multilabel Plate Reader.

\subsection{In vitro biofilm formation and treatment with antibiotics}

Starting from overnight cultures on blood agar plates, several colonies were re-suspended in saline $(0.9 \% \mathrm{w} / \mathrm{v} \mathrm{NaCl})$ to reach an optical density equal to 0.5 McFarland standard (Cobas Inocheck; Roche Diagnostics GmbH, Mannheim, Germany). This suspension was diluted 100 times in the appropriate growth medium used for biofilm formation (Table 1 ). Then, $200 \mu \mathrm{L}$ of the bacterial suspension was applied to each well of a flat-bottomed 96-well microtitre plate (Cellstar ${ }^{\circ}$; Greiner Bio-One, Frickenhausen, Germany). Plates were incubated statically for $24 \mathrm{~h}$ at $37^{\circ} \mathrm{C}$. Subsequently, the supernatants were discarded and the formed biofilms were washed once with $250 \mu \mathrm{L}$ of PBS. Biofilms were treated with $200 \mu \mathrm{L}$ of antibiotic solution (in $1 \%$ TSB, i.e. 100 times diluted in PBS) for $24 \mathrm{~h}$ at $37^{\circ} \mathrm{C}$. The applied concentrations of the antibiotics were $40 \mu \mathrm{g} / \mathrm{mL}$ for vancomycin, $50 \mu \mathrm{g} / \mathrm{mL}$ for teicoplanin, and $10 \mu \mathrm{g} / \mathrm{mL}$ for oxacillin, rifampicin and gentamicin.

\subsection{Evaluation of the biofilms}

The supernatants were discarded and the biofilms were washed twice with $250 \mu \mathrm{L}$ of PBS. The plates were stained with CV according to the method proposed by Stepanović et al. [9]. After fixing the biofilms with ethanol (96\%), the plates were dried and stained for $15 \mathrm{~min}$ with $230 \mu \mathrm{L}$ of Hucker's CV solution [0.5 mg CV (SigmaAldrich) dissolved in $5 \mathrm{~mL}$ of ethanol 100\%, and combined with $45 \mathrm{~mL}$ of an aqueous solution containing $1 \%$ ammonium oxalate (Sigma-Aldrich)]. The plates were washed to remove excess stain and were subsequently dried. Bound CV was eluted by adding $200 \mu \mathrm{L}$ of $5 \%$ acetic acid to each well. After $30 \mathrm{~min}$ of incubation at room temperature, $150 \mu \mathrm{L}$ of the eluate was transferred to a new 96-well plate and the absorbance of the eluate was measured at $590 \mathrm{~nm}$ using a VICTOR3 ${ }^{\mathrm{TM}}$ Multilabel Plate Reader.

For staining biofilms with XTT, the method described by Cerca et al. [8] was used. The XTT solution $(0.2 \mathrm{mg} / \mathrm{mL}$ XTT, $0.02 \mathrm{mg} / \mathrm{mL}$ phenazine methosulphate; Sigma-Aldrich) was prepared in $1 \% \mathrm{TSB}$, and incubation of the biofilms with the staining solution lasted for $2 \mathrm{~h}$ at $37^{\circ} \mathrm{C}$ in the dark. Absorbance of the supernatant was measured at $490 \mathrm{~nm}$ using a VICTOR3 ${ }^{\mathrm{TM}}$ Multilabel Plate Reader.

The results after antibiotic treatment of the biofilms were expressed relative to the staining result after treatment of the biofilm with the negative control (1\% TSB).

\subsection{Scanning electron microscopy (SEM)}

Biofilms were grown on glass coverslip disks (13 mm diameter; Assistent, Glaswarenfabrik Karl Hecht, Sondheim, Germany) for $24 \mathrm{~h}$ and were treated with antibiotics as mentioned previously. Biofilms on the coverslip disks were rinsed once with $1 \mathrm{~mL}$ of PBS and were fixed for $2 \mathrm{~h}$ with glutaraldehyde (2.5\%) in sodium cacodylate buffer $(0.1 \mathrm{M}, \mathrm{pH} 7.4)$ at room temperature. Afterwards, biofilms were washed with sodium cacodylate buffer and post-fixed for $2 \mathrm{~h}$ with osmium tetroxide ( $1 \%$ in Milli- $Q$ water) at $4{ }^{\circ} \mathrm{C}$, protected from light. The fixed samples were dehydrated with ethanol in ascending concentrations [30-50-70-90\% (v/v)] for $5 \mathrm{~min}$ and $100 \%(\mathrm{v} / \mathrm{v})$ ethanol for $3 \times 5 \mathrm{~min}$. For the final dehydration step, hexamethyldisilazane (HMDS) (Sigma-Aldrich) was used for $2 \times 15 \mathrm{~min}$. After overnight drying in a vacuum desiccator, the samples were sputter-coated with platinum for $120 \mathrm{~s}$ (Automatic sputter coater; Agar Scientific, Elektron Technology UK Ltd., Stansted, UK). Imaging was conducted on a JSM 7401F scanning electron microscope (JEOL, Tokyo, Japan). In each sample, three different areas were observed at magnifications of $1000 \times, 4000 \times$ and $10000 \times$.

\subsection{Statistical analysis}

Data were processed with Microsoft Excel (Microsoft Corp., Redmond, WA) and statistical analysis was conducted with IBM SPSS Statistics for Windows v.22.0 (IBM Corp., Armonk, NY). Differences between treatments were analysed by one-way analysis of variance (ANOVA), and differences between strains were characterised by two-way ANOVA. ANOVA analysis assumes that the data have a normal distribution and equal variances. Normality of the data was tested with the Shapiro-Wilk test and evaluation of the Q-Q plots. Equality of variances was analysed with the Levene's test. If the variances were not equal, Welch ANOVA analysis was applied. 
Table 1

Staphylococcus epidermidis strains used in the study.

\begin{tabular}{|c|c|c|c|}
\hline Strain & Description & Biofilm growth conditions & Reference \\
\hline $10 \mathrm{~b}$ & Clinical isolate; catheter-related BSI & $10 \% \mathrm{TSB}$ & UZ Leuven [14] \\
\hline 1457 & Clinical isolate; catheter-related BSI & $10 \% \mathrm{TSB}$ & [13] \\
\hline 567 & Clinical isolate; urinary tract catheter-related infection & $\mathrm{TSB}+4 \% \mathrm{NaCl}$ & [12] \\
\hline $12 \mathrm{c}$ & Clinical isolate; CVC subcutaneous section & TSB & UZ Leuven \\
\hline $13 c$ & Clinical isolate; CVC catheter tip & TSB & UZ Leuven \\
\hline $16 c$ & Clinical isolate; CVC catheter tip & TSB & UZ Leuven \\
\hline $17 \mathrm{c}$ & Clinical isolate; CVC subcutaneous section & TSB & UZ Leuven \\
\hline $22 c$ & Clinical isolate; CVC catheter tip & $10 \%$ TSB & UZ Leuven \\
\hline
\end{tabular}

BSI, bloodstream infection; TSB, tryptone soya broth; CVC, central venous catheter.

Table 2

Overview of minimum inhibitory concentrations (MICs) of planktonic cells of selected Staphylococcus epidermidis strains.

\begin{tabular}{llllll}
\hline \multirow{2}{*}{ Strain } & \multicolumn{4}{l}{ MIC $(\mu \mathrm{g} / \mathrm{mL})\left(\right.$ sensitivity $\left.^{\mathrm{a}}\right)$} & \\
\cline { 2 - 6 } & VAN & TEI & OXA & RIF & GEN \\
\hline 567 & $2(\mathrm{~S})$ & $1(\mathrm{~S})$ & $0.25(\mathrm{~S})$ & $0.25(\mathrm{~S})$ & $0.25(\mathrm{~S})$ \\
1457 & $1(\mathrm{~S})$ & $4(\mathrm{~S})$ & $0.25(\mathrm{~S})$ & $0.25(\mathrm{~S})$ & $0.25(\mathrm{~S})$ \\
$10 \mathrm{~b}$ & $1(\mathrm{~S})$ & $4(\mathrm{~S})$ & $0.25(\mathrm{~S})$ & $0.25(\mathrm{~S})$ & $1(\mathrm{~S})$ \\
\hline
\end{tabular}

VAN, vancomycin; TEI, teicoplanin; OXA, oxacillin; RIF, rifampicin; GEN, gentamicin; $\mathrm{S}$, sensitive.

a Determined following European Committee on Antimicrobial Susceptibility Testing (EUCAST) clinical breakpoints for staphylococci.

When the ANOVA analysis was significant, and the differences between the tested groups were significant, multiple $t$-tests were conducted to locate the differences. To correct for multiple testing, the significance level was adjusted according to Šídák. Overall, a significance level of $P<0.05$ was presumed.

\section{Results}

\subsection{Minimum inhibitory concentrations}

For S. epidermidis strains 567, 1457 and 10b, antibiotic susceptibilities were determined according the EUCAST guidelines (Table 2). According to EUCAST breakpoints (http://www.eucast.org), all of the strains were sensitive to the tested antibiotics. The antibiotic sensitivities of the five other strains (S. epidermidis 12c, 13c, 16c, 17c and 22c) were determined by VITEK ${ }^{\circledR} 2$ analysis (bioMérieux Benelux s.a./n.v., Brussels, Belgium). These strains were also found to be sensitive to the selected antibiotics (data not shown).

\subsection{Assessment of the effects of antibiotics on in vitro biofilms}

To test the effects of the antibiotics on S. epidermidis biofilms, five antibiotics with different mechanisms of action were used. Vancomycin, teicoplanin, oxacillin and rifampicin were selected because they are the antibiotics of choice for the treatment of device-related infections with S. epidermidis. The effects of gentamicin were tested as this antibiotic is used in temporary spacers following the removal of infected prosthetic devices [17]. The applied concentrations corresponded to the peak serum concentrations of the antibiotics [18-20].

Fig. 1 shows the results of CV staining after treatment of 24-h-old biofilms with the respective antibiotics. Surprisingly, vancomycin and teicoplanin increased the total biofilm biomass in all of the tested strains relative to treatment of the biofilms with $1 \%$ TSB. Rifampicin was the only antibiotic resulting in a decrease of biofilm biomass produced by S. epidermidis $12 \mathrm{c}, 13 \mathrm{c}, 1457$ and 22c. Oxacillin and gentamicin were marginally effective in reducing the biofilm biomass.

Fig. 2 shows the bacterial viability after treatment with antibiotics as assayed by XTT staining. In contrast to the effect on

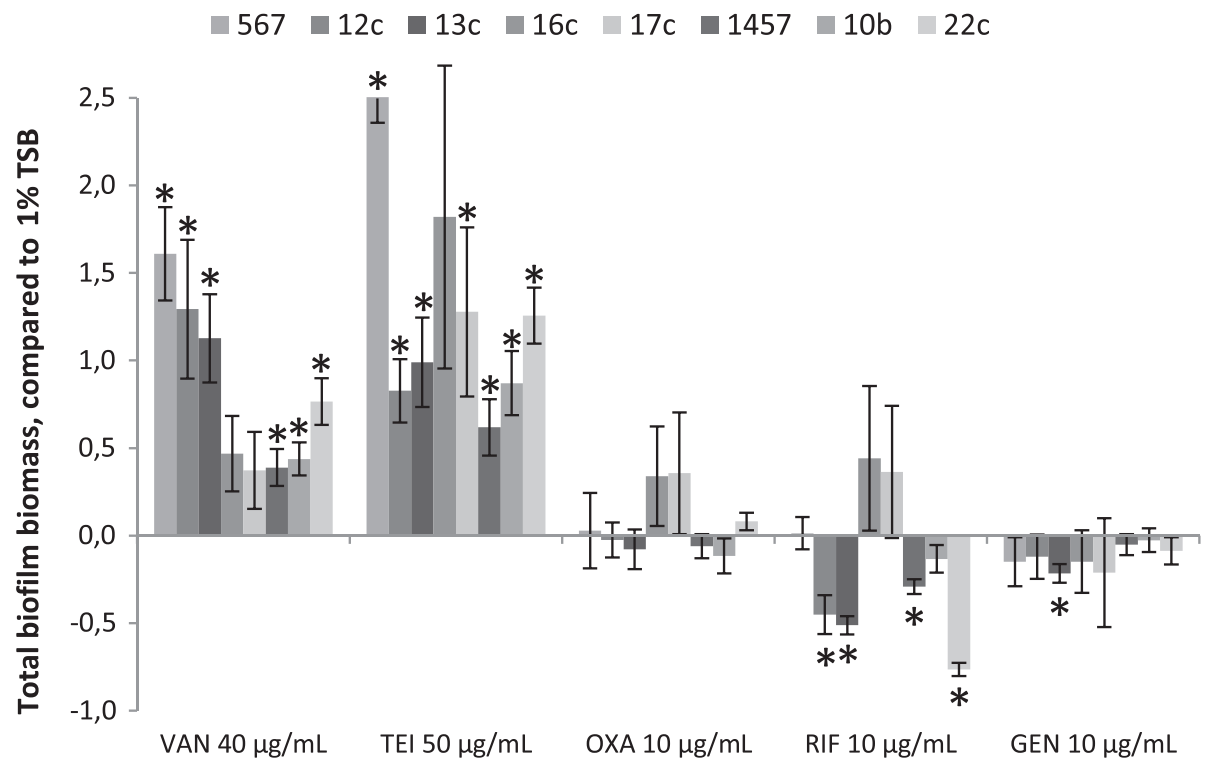

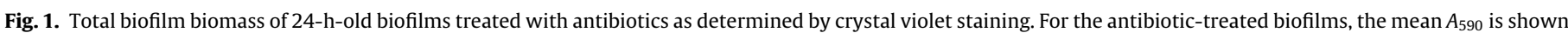

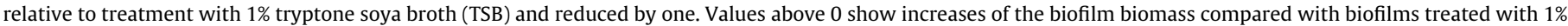

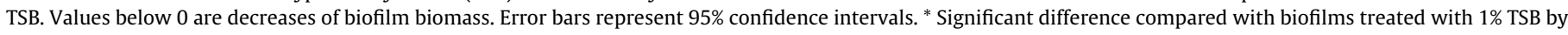
one-way ANOVA $(P<0.05)$. VAN, vancomycin; TEI, teicoplanin; OXA, oxacillin; RIF, rifampicin; GEN, gentamicin. 


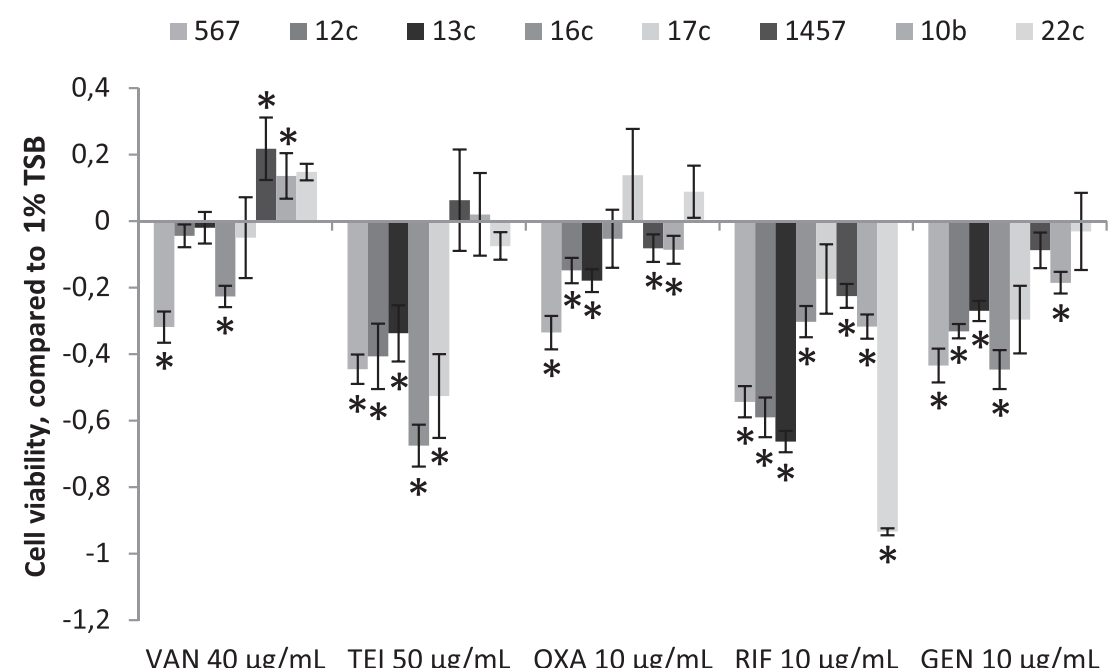

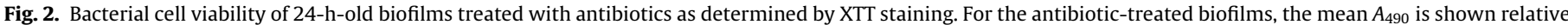

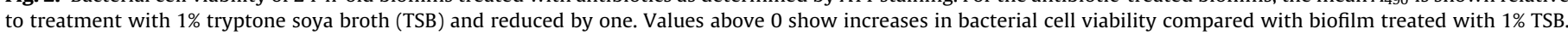

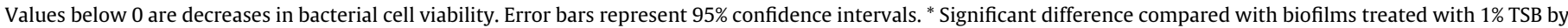
one-way ANOVA $(P<0.05)$. VAN, vancomycin; TEI, teicoplanin; OXA, oxacillin; RIF, rifampicin; GEN, gentamicin.

total biomass, the effects of the antibiotics on bacterial viability were more pronounced. Still, a difference was seen between the killing activity of the glycopeptides in comparison with the other antibiotics. Vancomycin in particular displayed a low potential to kill biofilm-associated bacteria. Moreover, treatment of biofilms produced by S. epidermidis 1457 and 10b significantly increased biofilm-associated viability. Teicoplanin was unable to affect the bacterial viability in biofilms produced by S. epidermidis 1457 , $10 \mathrm{~b}$ and 22c. Most probably these observations are related to the strong biofilm-producing potential of these strains. Treatment of the biofilms with the other antibiotics killed a substantial amount of biofilm-related bacteria, with rifampicin displaying the highest killing activity (Fig. 2).

SEM was performed after antibiotic treatment of 24-h-old $S$. epidermidis 1457 biofilms. Figs. 3 and 4 show SEM images at magnifications of $1000 \times, 4000 \times$ and $10000 \times$. Although difficult to quantify, treatment with $40 \mu \mathrm{g} / \mathrm{mL}$ vancomycin (Fig. 3C) showed a slight increase in biofilm accumulation compared with $1 \%$ TSB (Fig. 3A). In contrast to this observation, the images could not confirm increased biofilm accumulation after treatment with $50 \mu \mathrm{g} / \mathrm{mL}$ teicoplanin (Fig. 3E), even though staining of the biofilms with $\mathrm{CV}$ showed an increase in total biofilm biomass, as was the case for vancomycin treatment (Fig. 1). Notably, biofilms treated with the glycopeptides contained damaged bacteria (yellow frames in Fig. 3D and F), whilst these were not visible in non-treated biofilms (Fig. 3B). Treatment with $10 \mu \mathrm{g} / \mathrm{mL}$ oxacillin (Fig. 4C and D) resulted in fewer bacteria, but a matrix or bacterial debris remained attached to the surface. Treatment with $10 \mu \mathrm{g} / \mathrm{mL}$ rifampicin (Fig. 4E and F) visibly decreased the bacterial load and the amount of matrix. Finally, $10 \mu \mathrm{g} / \mathrm{mL}$ gentamicin (Fig. 4G and $\mathrm{H}$ ) did not display a clear effect on the biofilm.

\subsection{Evaluation of the effects of antibiotics combined with rifampicin on in vitro Staphylococcus epidermidis biofilms}

Biofilms produced by S. epidermidis 567, 1457 and 10b were treated with combinations of antibiotic and rifampicin. Results of the CV and XTT staining are shown in Figs 5 and 6, respectively. The combination of vancomycin or teicoplanin plus rifampicin appeared to be significantly more effective in reducing the biofilm biomass (Fig. 5) and biofilm-associated viability (Fig. 6) than vancomycin or teicoplanin alone. Combining rifampicin with oxacillin or gentamicin did not influence the activity on the biofilm biomass (Fig. 5), but for oxacillin the biofilm-associated viability significantly decreased in comparison to treatment with oxacillin alone (Fig. 6).

\section{Discussion}

Biofilm-related infections are becoming more important in clinical practice with the increasing use of implanted medical devices. In addition, bacteria growing in a biofilm are characterised by higher resistance towards antibiotics, as shown by determination of the biofilm MICs and MBCs as well as MBECs [7]. Therefore, routine determination of bacterial antibiotic susceptibility during planktonic growth is not a good prediction for the response of biofilm-associated bacteria. Until now, methods to evaluate the effects of antibiotics on biofilms in routine clinical laboratories have been missing. In this study, CV and XTT staining were used to assess the effects of antibiotics on S. epidermidis biofilms in vitro. CV is widely used to evaluate bacterial biofilm formation. However, it gives no information about the viability of bacteria and the relative amount of bacteria versus matrix. Thus, we combined CV with XTT staining to additionally assess bacterial viability [8]. This methodology allows evaluation of the biofilm biomass and bacterial viability separately and has been applied previously. The results of previous studies showed that biofilm-associated bacteria are less sensitive to killing by antibiotics compared with their planktonic counterparts [8] and that the extracellular matrix remained attached to the surface, constituting a risk for recolonisation [6,21]. However, in the present study, we have shown that treatment of biofilms with vancomycin and teicoplanin increased the total biofilm biomass. Although SEM images of vancomycin-treated biofilms showed a small increase in bacterial accumulation (Fig. 3C and D), teicoplanin did not obviously increase the amount of biofilm-associated bacteria (Fig. 3E and F). Yet both glycopeptides caused visible damage to the bacteria incorporated in the biofilm. The increased amount of biofilm-associated bacteria might be an explanation for the observed rise in the biofilm biomass (Fig. 1).

Assessment of bacterial viability demonstrated a low killing efficacy of vancomycin on biofilm-associated S. epidermidis, even though the planktonic bacteria were sensitive to vancomycin. The same observation was made by Monzón et al. [18] who tested the efficacy of vancomycin on S. epidermidis biofilms by bacterial 

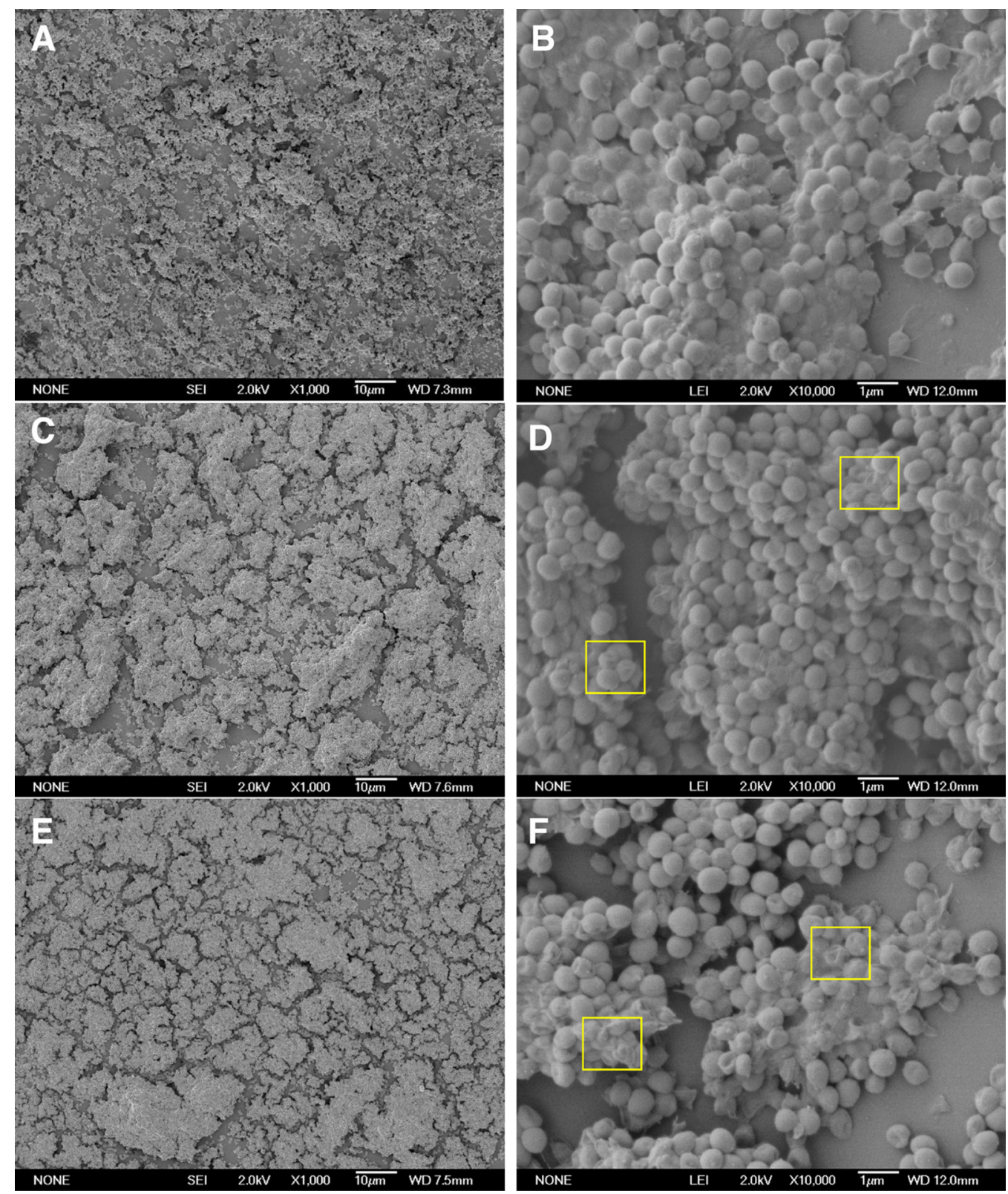

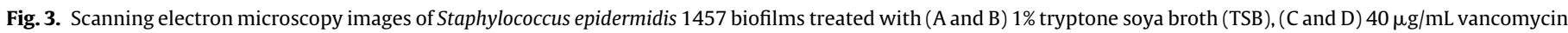
and (E and F) $50 \mu \mathrm{g} / \mathrm{mL}$ teicoplanin. Images at magnifications of $1000 \times$ (left) and $10000 \times$ (right) are shown. Yellow frames mark groups of damaged bacteria.

viability determination with an ATP bioluminescence assay. The lower efficacy of vancomycin was attributed to the extracellular biofilm matrix. Furthermore, Mathur et al. [22] demonstrated that the MICs and killing efficacies of vancomycin and teicoplanin on planktonic S. epidermidis bacteria were negatively influenced by the addition of extracted extracellular biofilm matrix. They hypothesised that the glycopeptides were inactivated by binding matrix components [22]. However, it is often assumed that the extracellular biofilm matrix acts as a physical barrier complicating the diffusion of antimicrobial agents into the biofilm. The hypothesis of decreased penetration of vancomycin into the biofilm is contradicted by the observation that vancomycin was present in the biofilm at concentrations exceeding its MIC and MBC values [23]. Both sequestration and decreased penetration could explain the decreased efficacy of vancomycin observed in the current study.

The results of XTT staining in addition suggested a relationship between the response to treatment with vancomycin and teicoplanin and the biofilm-producing capacity of the bacterial strain. S. epidermidis 1457 and 10b, which are better biofilm producers than $S$. epidermidis 567, were less prone to killing by the glycopeptides. This observation is consistent with the conclusions made by Antunes et al. [24], who investigated the MBEC of vancomycin on biofilms produced by $S$. epidermidis strains isolated from CVCs and observed that the stronger biofilm producers displayed a higher MBEC [24]. These observations could be explained by the 'inoculum effect', which means that for some antimicrobial agents the effectiveness is related to the bacterial cell density of the inoculum. The antibiotic becomes less effective when the bacterial cell density of the inoculum is higher because a lower amount of antibiotic molecules is available per bacterial cell [25]. The relationship between the killing activities of vancomycin and teicoplanin and the biofilm-producing capacity of the strain is then attributable to a higher bacterial density in biofilms produced by the 'strong' biofilm-producing strains. In addition, teicoplanin was found to be more effective in killing biofilm-associated bacteria than vancomycin at peak serum concentrations (Fig. 2). 

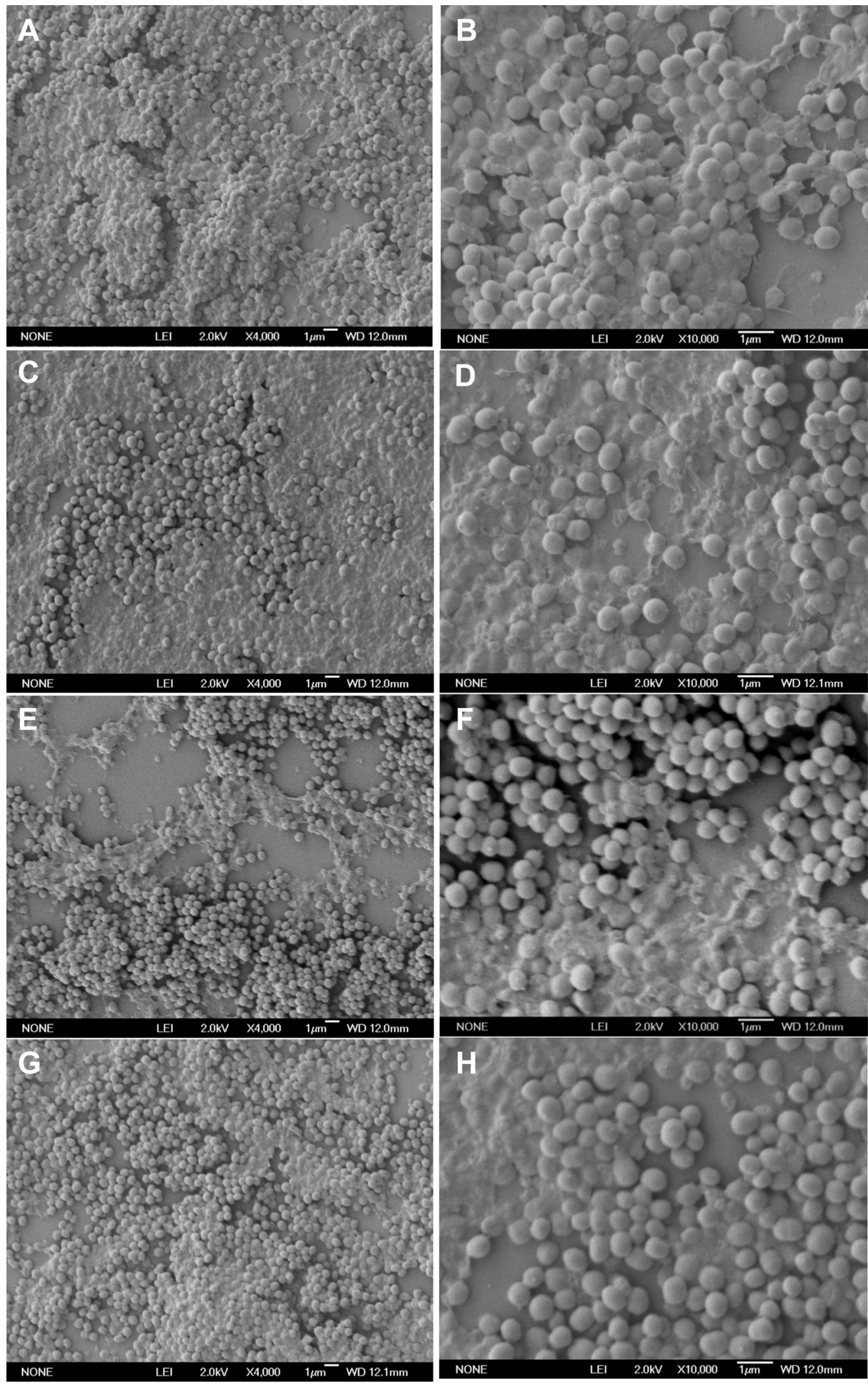

Fig. 4. Scanning electron microscopy images of Staphylococcus epidermidis 1457 biofilms treated with (A and B) $1 \%$ tryptone soya broth (TSB), (C and D) $10 \mu \mathrm{g} / \mathrm{mL}$ oxacillin, (E and F) $10 \mu \mathrm{g} / \mathrm{mL}$ rifampicin and $(\mathrm{G}$ and $\mathrm{H}) 10 \mu \mathrm{g} / \mathrm{mL}$ gentamicin. Images at magnifications of $4000 \times$ (left) and $10000 \times$ (right) are shown. 


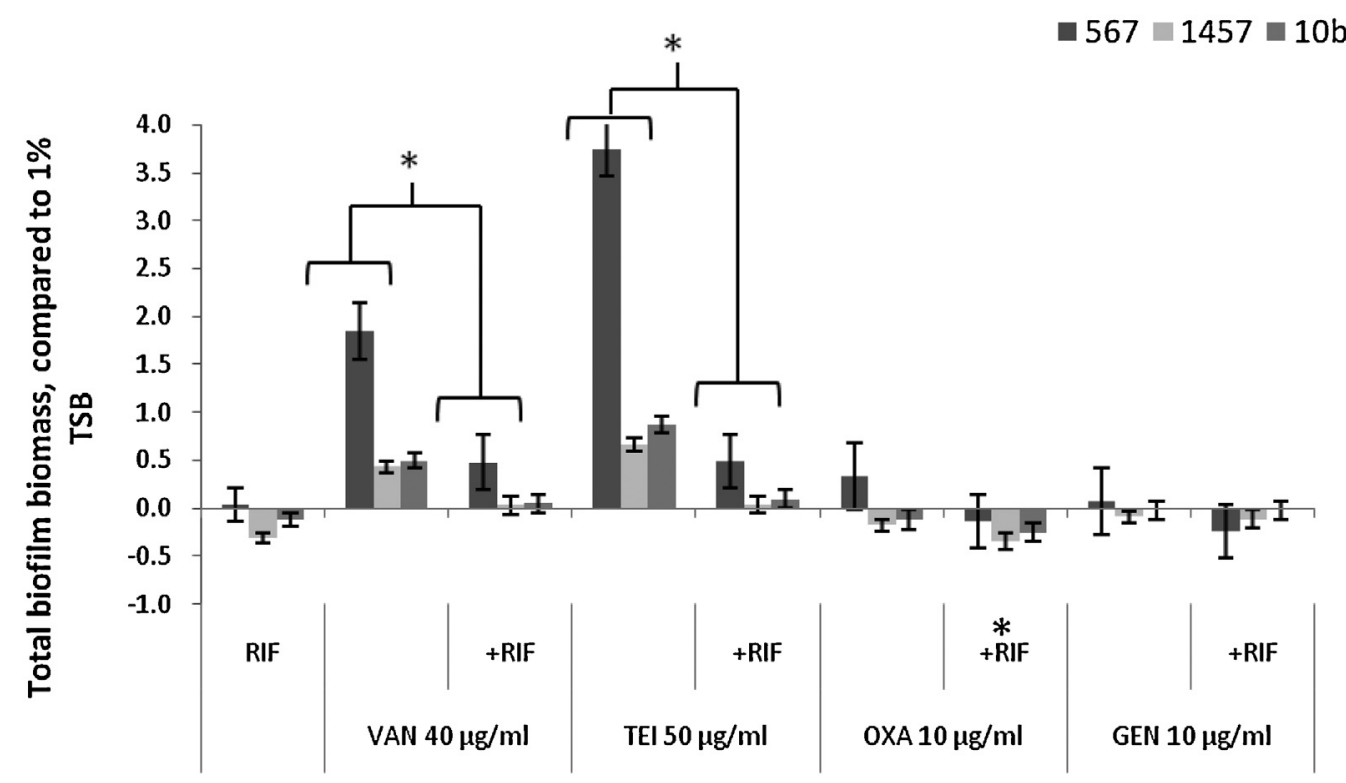

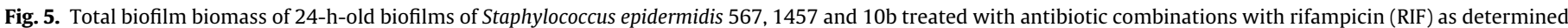

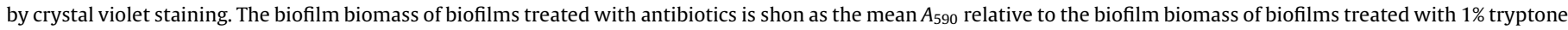

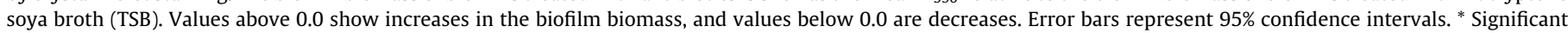

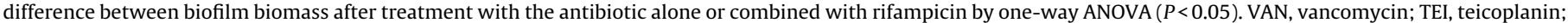
OXA, oxacillin; GEN, gentamicin.

In clinical practice, treatment of foreign body-associated infections often consists of a combination of a glycopeptide with rifampicin. In vitro assessment of the effects of glycopeptide/rifampicin combinations on $S$. epidermidis biofilms demonstrated that the increase in total biofilm biomass after treatment of the biofilms with teicoplanin or vancomycin alone was neutralised (Fig. 5). The killing efficacies of antibiotic combinations with rifampicin were improved compared with the antibiotics alone (Fig. 6). These observations give evidence for the combined strategy in dealing with biofilm-associated infections. As in the literature, the in vitro results in the current study advised against the combination of rifampicin with gentamicin [26].
The observed inefficacy of vancomycin to kill biofilm-associated bacteria in vitro may have consequences in vivo. Indeed, vancomycin was tested in different animal models of device-related infections and failed to eradicate the biofilm [27-29]. The concentration of vancomycin detected in the biofilms was above the MIC values [29], but only cellular structures were destroyed and matrix was left [28]. The remaining material after antibiotic treatment could function as anchorage for other bacteria and encourage re-growth of the biofilm and re-colonisation of the device [6].

In accordance with our suggestion that $50 \mu \mathrm{g} / \mathrm{mL}$ teicoplanin is more effective in killing biofilm-associated bacteria than $40 \mu \mathrm{g} / \mathrm{mL}$ vancomycin (Fig. 2), Del Pozo et al. [30] observed the same trend in

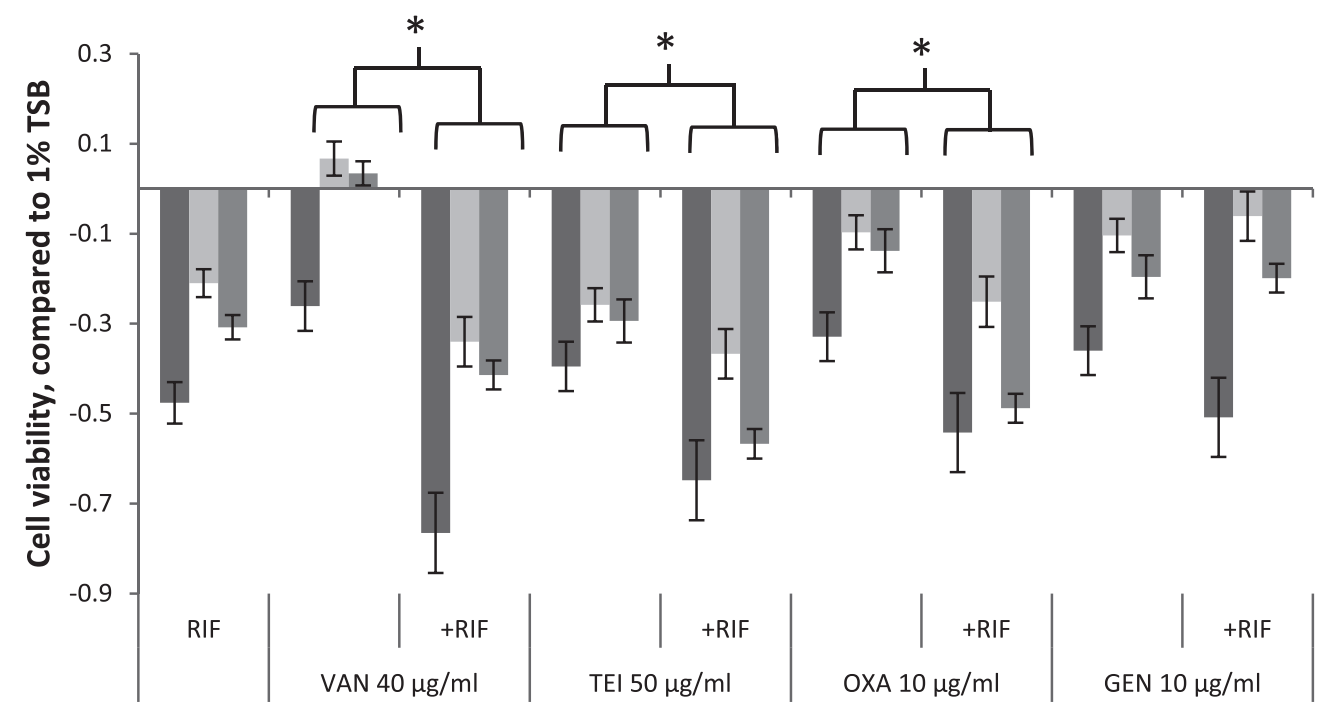

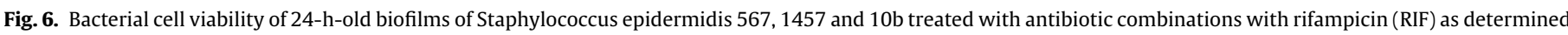

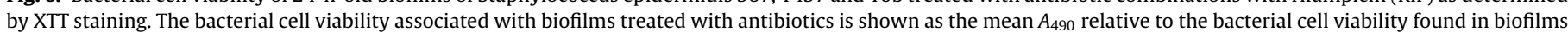

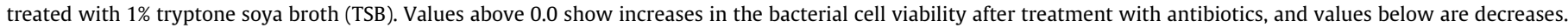

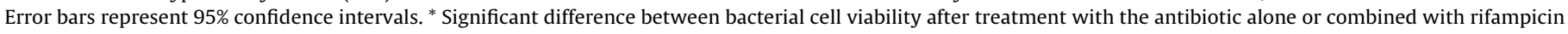
by one-way ANOVA $(P<0.05)$. VAN, vancomycin; TEI, teicoplanin; OXA, oxacillin; GEN, gentamicin. 
a clinical trial of antibiotic-lock therapy in catheter-related bloodstream infections.

In conclusion, assessing the effects of antibiotics by CV and XTT staining plus microscopy showed an increased biofilm biomass and decreased killing efficacies of the glycopeptides, in particular vancomycin, on biofilm-associated bacteria. This might be explained by the negative impact of the extracellular biofilm matrix on the biological activity of the glycopeptides Also, an inverse correlation between vancomycin susceptibility and biofilm-forming capacity was found.

\section{Funding}

This work was conducted with the financial support of the Flemish Agency for Innovation by Science and Technology (IWT) Grant number: 093256.

\section{Competing interests}

None declared.

\section{Ethical approval}

Not required.

\section{References}

[1] McDougald D, Rice SA, Barraud N, Steinberg PD, Kjelleberg S. Should we stay or should we go: mechanisms and ecological consequences for biofilm dispersal. Nat Rev Microbiol 2012;10:39-50.

[2] Bjarnsholt T. The role of bacterial biofilms in chronic infections. APMIS Suppl 2013;121:1-51.

[3] Yao Y, Sturdevant DE, Otto M. Genomewide analysis of gene expression in Staphylococcus epidermidis biofilms: insights into the pathophysiology of S. epidermidis biofilms and the role of phenol-soluble modulins in formation of biofilms. J Infect Dis 2005;191:289-98.

[4] Stewart PS. Mechanisms of antibiotic resistance in bacterial biofilms. Int J Med Microbiol 2002;292:107-13.

[5] Høiby N, Bjarnsholt T, Givskov M, Molin S, Ciofu O. Antibiotic resistance of bacterial biofilms. Int J Antimicrob Agents 2010;35:322-32.

[6] Skogman ME, Vuorela PM, Fallarero A. Combining biofilm matrix measurements with biomass and viability assays in susceptibility assessments of antimicrobials against Staphylococcus aureus biofilms. J Antibiot (Tokyo) 2012;65:453-9.

[7] Qu Y, Daley AJ, Istivan TS, Rouch DA, Deighton MA. Densely adherent growth mode, rather than extracellular polymer substance matrix build-up ability, contributes to high resistance of Staphylococcus epidermidis biofilms to antibiotics. J Antimicrob Chemother 2010;65:1405-11.

[8] Cerca N, Martins S, Cerca F, Jefferson KK, Pier GB, Oliveira R, et al. Comparative assessment of antibiotic susceptibility of coagulase-negative staphylococci in biofilm versus planktonic culture as assessed by bacterial enumeration or rapid XTT colorimetry. J Antimicrob Chemother 2005;56:331-6.

[9] Stepanović S, Vuković D, Hola V, Di Bonaventura G, Djukić S, Cirković I, et al. Quantification of biofilm in microtiter plates: overview of testing conditions and practical recommendations for assessment of biofilm production by staphylococci. APMIS 2007;115:891-9.

[10] Hsu CY, Lin MH, Chen CC, Chien SC, Cheng YH, Su IN, et al. Vancomycin promotes the bacterial autolysis, release of extracellular DNA, and biofilm formation in vancomycin-non-susceptible Staphylococcus aureus. FEMS Immunol Med Microbiol 2011;63:236-47.
[11] Cargill JS, Upton M. Low concentrations of vancomycin stimulate biofilm formation in some clinical isolates of Staphylococcus epidermidis. J Clin Pathol 2009;62:1112-6.

[12] Batzilla CF, Rachid S, Engelmann S, Hecker M, Hacker J, Ziebuhr W. Impact of the accessory gene regulatory system (Agr) on extracellular proteins, codY expression and amino acid metabolism in Staphylococcus epidermidis. Proteomics 2006;6:3602-13

[13] Mack D, Siemssen N, Laufs R. Parallel induction by glucose of adherence and a polysaccharide antigen specific for plastic-adherent Staphylococcus epidermidis: evidence for functional relation to intercellular adhesion. Infect Immun 1992:60:2048-57.

[14] Van Wijngaerden E, Peetermans WE, Vandersmissen J, Van Lierde S, Bobbaers $\mathrm{H}$, Van Eldere J. Foreign body infection: a new rat model for prophylaxis and treatment. J Antimicrob Chemother 1999;44:669-74.

[15] Rachid S, Ohlsen K, Witte W, Hacker J, Ziebuhr W. Effect of subinhibitory antibiotic concentrations on polysaccharide intercellular adhesin expression in biofilm-forming Staphylococcus epidermidis. Antimicrob Agents Chemother 2000;44:3357-63.

[16] European Committee for Antimicrobial Susceptibility Testing (EUCAST) of the European Society of Clinical Microbiology and Infectious Diseases (ESCMID). Determination of minimum inhibitory concentrations (MICs) of antibacterial agents by broth dilution. Clin Microbiol Infect 2003;9:ix-XV.

[17] Donati D, Biscaglia R. The use of antibiotic-impregnated cement in infected reconstructions after resection for bone tumours. J Bone Joint Surg $\mathrm{Br}$ 1998; 80:1045-50.

[18] Monzón M, Oteiza C, Leiva J, Lamata M, Amorena B. Biofilm testing of Staphylococcus epidermidis clinical isolates: low performance of vancomycin in relation to other antibiotics. Diagn Microbiol Infect Dis 2002;44:319-24.

[19] Demczar DJ, Nafziger AN, Bertino JS. Pharmacokinetics of gentamicin at traditional versus high doses: implications for once-daily aminoglycoside dosing. Antimicrob Agents Chemother 1997;41:1115-9.

[20] Yufang $H$, Zhaohui Z, Huabin Z, Lijuan L, Shouzhuo Y. A sensitive and selective sensor-coated molecularly imprinted sol-gel film incorporating $\beta$-cyclodextrin-multi-walled carbon nanotubes and cobalt nanoparticleschitosan for oxacillin determination. Surf Interface Anal 2012;44:334-41.

[21] Toté K, Vanden Berghe D, Deschacht M, de Wit K, Maes L, Cos P. Inhibitory efficacy of various antibiotics on matrix and viable mass of Staphylococcus aureus and Pseudomonas aeruginosa biofilms. Int J Antimicrob Agents 2009;33:525-31.

[22] Mathur T, Singhal S, Khan S, Upadhyay D, Fatma T, Rattan A. Adverse effect of staphylococci slime on in vitro activity of glycopeptides. Jpn J Infect Dis 2005;58:353-7.

[23] Darouiche RO, Dhir A, Miller AJ, Landon GC, Raad II, Musher DM. Vancomycin pepentration into biofilm covering infected prostheses and effect on bacteria. J Infect Dis 1994;170:720-3.

[24] Antunes ALS, Bonfanti JW, Perez LRR, Pinto CC, Freitas AL, Macedo AJ, et al. High vancomycin resistance among biofilms produced by Staphylococcus species isolated from central venous catheters. Mem Inst Oswaldo Cruz 2011;106: 51-5.

[25] Udekwu K, Parrish N, Ankomah P, Baquero F, Levin B. Functional relationship between bacterial cell density and the efficacy of antibiotics. J Antimicrob Chemother 2009;63:745-57.

[26] Gagnon RF, Richards GK, Wiesenfeld L. Staphylococcus epidermidis biofilms: unexpected outcome of double and triple antibiotic combinations with rifampin. ASAIO Trans 1991;37:M158-60.

[27] He NA, Hu J, Liu HY, Zhu T, Huang B, Wang X, et al. Enhancement of vancomycin activity against biofilms by using ultrasound-targeted microbubble destruction. Antimicrob Agents Chemother 2011;55:5331-7.

[28] Aybar Y, Ozaras R, Besirli K, Engin E, Karabulut E, Salihoglu T, et al. Efficacy of tigecycline and vancomycin in experimental catheter-related Staphylococcus epidermidis infection: microbiological and electron microscopic analysis of biofilm. Int J Antimicrob Agents 2012;39:338-42.

[29] Isiklar ZU, Darouiche RO, Landon GC, Beck T. Efficacy of antibiotics alone for orthopaedic device related infections. Clin Orthop Relat Res 1996;332: 184-9.

[30] Del Pozo JL, García Cenoz M, Hernáez S, Martínez A, Serrera A, Aguinaga A, et al. Effectiveness of teicoplanin versis vancomycin lock therapy in the treatment of port-related coagulase-negative staphylococci bacteraemia: a prospective case-series analysis. Int J Antimicrob Agents 2009;34:482-5. 